\title{
Predicting cardiac surgery-associated acute kidney injury: The CRATE score
}

\author{
Pablo Jorge-Monjas, $\mathrm{C}^{\mathrm{a}}$, Juan Bustamante-Munguira, $\mathrm{MD}, \mathrm{PhD}^{\mathrm{b}, *}$, Mario Lorenzo, $\mathrm{MD}, \mathrm{PhD}{ }^{\mathrm{a}}$, \\ María Heredia-Rodríguez, MD, PhD a,c Inmaculada Fierro, PhD ${ }^{\mathrm{d}}$, Esther Gómez-Sánchez, MD, PhD ${ }^{\text {a,c }}$, \\ Alfonso Hernandez, MD a, Francisco J. Álvarez, MD, PhD d, Jesús F. Bermejo-Martin, MD, PhD c,e, \\ Estefanía Gómez-Pesquera, MD, PhD a , José I. Gómez-Herreras, MD, PhD a,c , Eduardo Tamayo, MD, PhD a,c \\ a Department of Anesthesiology and Reanimation, Hospital Clinico Universitario de Valladolid, Valladolid, Spain \\ b Department of Cardiovascular Surgery, Hospital Universitario de La Princesa, Madrid, Spain \\ c Immunity, Risk of Infection and Sepsis group (IRIS), Hospital Clínico Universitario de Valladolid, Valladolid, Spain \\ ${ }^{\mathrm{d}}$ Department of Pharmacology and Therapeutics, Valladolid University Physicians College, Valladolid, Spain \\ e Infection E' Immunity Medical Investigation group, Hospital Clínico Universitario-IECSCYL, Valladolid, Spain
}

\section{A R T I C L E I N F O}

\section{Keywords:}

Cardiac surgery

Renal insufficiency

Risk prediction

Risk score

\begin{abstract}
A B S T R A C T
Purpose: Acute kidney injury (AKI) is a frequent complication after cardiac surgery and is associated with increased mortality. The aim was to design a nondialytic AKI score in patients with previously normal renal function undergoing cardiac surgery.

Methods: Data were collected on 909 patients who underwent cardiac surgery with cardiopulmonary bypass between 2012 and 2014. A total of 810 patients fulfilled the inclusion criteria. Patients were classified as having AKI based on the RIFLE criteria. Postoperative AKI occurred in 137 patients (16.9\%). Several parameters were recorded preoperatively, intraoperatively, and at intensive care unit admission, looking for a univariate and multivariate association with AKI risk. A second data set of 741 patients, from 2 different hospitals, was recorded as a validation cohort. Results: Four independent risk factors were included in the CRATE score: creatinine (odds ratio [OR], 9.66; 95\% confidence interval [CI], 4.77-19.56; $P<.001$ ), EuroSCORE (OR, 1.40; CI, 1.29-1.52; $P<.001$ ), lactate (OR, 1.03; CI, 1.01$1.04 ; P<.001)$, and cardiopulmonary bypass time (OR, $1.01 ; \mathrm{CI}, 1.01-1.02 ; P<.001)$. The accuracy of the model was good, with an area under the curve of 0.89 ( $\mathrm{Cl}, 0.85-0.92)$. The CRATE score retained good discrimination in validation cohort, with an area under the curve of 0.81 (95\% CI, 0.78-0.85).

Conclusions: CRATE score is an accurate and easy to calculate risk score that uses affordable and widely available variables in the routine care surgical patients.
\end{abstract}

(c) 2015 Published by Elsevier Inc.

\section{Introduction}

Acute kidney injury (AKI) is a frequent and severe complication after cardiac surgery, being this kind of surgery the second most common cause of AKI in the intensive care unit (ICU) [1]. In this context and depending on the definition used, AKI could affect up to $39 \%$ of patients undergoing cardiac surgery procedures, with $1 \%$ to $5 \%$ needing renal replacement therapy (RRT) [2]. Patients developing AKI have increased morbidity and worse surviving rates than those with normal renal function. In addition, it increases ICU and inhospital stay, risk of infection, and hospitalization cost [3]. Therefore, AKI is an important complication closely related to cardiac surgery using cardiopulmonary bypass (CPB). This particular relation gave rise to the appearance of the term cardiac surgery-associated acute kidney injury (CSA-AKI) [4].

\footnotetext{
* Corresponding author at: Department of Cardiovascular Surgery, Hospital Universitario de La Princesa, Madrid, Spain. Tel.: + 34 915202268; fax: + 34915202201.

E-mail address: jbustamantemunguira@gmail.com (J. Bustamante-Munguira).
}

Risk stratification has become essential in cardiac surgical practice, specifically focused in mortality (ie, EuroSCORE and ACEF score) [5]. Because of the implications of CSA-AKI in outcome, this clinical entity has been targeted in risk stratification models. Previously, some authors have developed several algorithms trying to predict renal failure not requiring dialysis [6]. The Multicenter Study of Perioperative Ischemia Research Group (MCSPI) model proposed by Aronson et al [7] included patients who underwent coronary artery bypass grafting (CABG) surgery, evaluating preoperative and intraoperative variables. Brown et al [8] produced an algorithm, the Northern New England Cardiovascular Disease Study Group (NNECDSG) score, with preoperative characteristics that included patients scheduled for CABG. None of these models take into account the early postoperative period, which is considered by some authors as the best time to apply a cardiac surgery-specific score because of the influence of intraoperative variables in the clinical course of these patients [9]. Therefore, it would be necessary to use a clinical tool estimating the risk by using preoperative, intraoperative, and postoperative variables. Palomba et al [9] followed up 603 patients 
after CABG, valvular surgery, or both, developing the acute kidney injury following cardiac surgery (AKICS) score. They added postoperative period data (central venous pressure and low cardiac output) to preoperative and intraoperative items in their model [9]. However, these previous 3 scores because of the AKI definition used may not predict less severe cases of AKI, which was previously shown as an important factor in morbidity and mortality $[2,10,11]$.

Our hypothesis is that using AKI risk factors from the preoperative and intraoperative period and adding organ function assessment variables obtained in the early postoperative period, it would be possible to create an accurate score for predicting AKI risk. The aim of this study was to design a nondialytic AKI risk score, including less severe cases, in patients with previously normal renal function undergoing cardiac surgery with $\mathrm{CPB}$.

\section{Materials and methods}

\subsection{Study design}

A prospective open cohort study was designed to assess risk factors for AKI after cardiac surgery with CPB, carried out between January 2012 and January 2014 in the Clinic University Hospital, Valladolid (Spain), a level III health care medical center with 800 beds. Patients were classified as having CSA-AKI based on the RIFLE criteria [3] using the peak postoperative plasma creatinine in the ICU. For baseline creatinine, we used that of hospital admission. Patients who met the RIFLE criteria for CSA-AKI (Risk, Injury and Failure) were classified as "AKI," whereas those who did not were classified as "NON-AKI." Those patients who required RRT (RIFLE class L or E) were classified into F category to avoid losing cases. Staging these patients in F class can improve the predictive value of this system [12].

The Valladolid Clinic University Hospital Ethics Committee approved the study and waived the need for an informed consent of the patients, who, however, gave their written consent to the storage in the hospital database for scientific treatment of their data in an anonymous form at the time of the hospital admission, according to the Spanish law regulating personal privacy matters.

The validation cohort consisted of consecutive cases at the Valladolid Clinic University Hospital and at La Princesa University Hospital between March 2014 and March 2015. This validation process evaluated the generalizability based on a differing geographic data collection and applied to a different period [13].

\subsection{Study population}

During the 3-year period of the study, adult patients ( 18 years or older) with normal renal function, scheduled for CABG and/or valve surgery with $\mathrm{CPB}$, were included. Transplant patients, patients with any degree of renal insufficiency (acute or chronic), those who died in the first 24 hours after the surgery, and patients undergoing off-pump cardiac procedures were excluded. A total of 1551 patients from 2 different institutions were included. A total of 810 patients were enrolled consecutively as a score development cohort, whereas the validation cohort consisted of 741 patients.

\subsection{Intraoperative care and anesthesia}

Induction of anesthesia was initiated with intravenous midazolam and etomidate. Rocuronium was used to achieve neuromuscular blockade. The lungs were ventilated with an oxygen/air mixture to maintain normocapnia. Sevoflurane, midazolam, and fentanyl were used in combination for maintenance of anesthesia. A radial, brachial, or femoral artery catheter was inserted for measurement of arterial blood pressure and blood sampling; and a trilumen internal jugular catheter, for measurement of central venous pressure, blood sampling, and fluid/medication administration. Temperature was obtained with an esophageal probe. Urine output was measured with a Foley catheter. Five-lead electrocardiogram was monitored continuously. Cardiopulmonary bypass with systemic heparinization $(350 \mathrm{U} / \mathrm{kg}$ ) was started. Additional heparin boluses were administered to maintain an activated clotting time greater than 400 seconds. Pump flows from 45 to $55 \mathrm{~mL} / \mathrm{kg}$ per minute, moderate systemic hypothermia $\left(28^{\circ} \mathrm{C}-30^{\circ} \mathrm{C}\right)$, and intermittent cold blood cardioplegia were used for most patients. The mean arterial pressure was maintained between 60 and $80 \mathrm{~mm} \mathrm{Hg}$. Heparin was reversed with protamine at a 1.5:1 ratio after completion of CPB and removal of cannulae.

\subsection{Postoperative care}

When surgery was finished, patients were transferred to the ICU, where they were treated according to a standard regimen. Hemodynamic values were assessed at heart rate at 70 to 80 beats per minute and mean arterial pressure at 65 to $80 \mathrm{~mm} \mathrm{Hg}$, using vasopressor and/or inotropic support depending on individual status of the patient. Basic intravenous fluid administration consisted of $0.9 \%$ $\mathrm{NaCl}$, and gelatin was infused if needed. Fluid balance, rectal temperature, peripheral temperature (axillary), and drainage output were recorded every hour. Mechanical ventilation was performed with $50 \%$ oxygen using volume-controlled ventilation and tidal volume of 6 to 8 $\mathrm{mL} / \mathrm{kg}$ with $5 \mathrm{~cm} \mathrm{H}_{2} \mathrm{O}$ of positive end-expiratory pressure. Sedation was provided with propofol and analgesia with morphine boluses as required. Samples of arterial blood gases were analyzed by standard techniques using an automated analyzer at anesthesia induction and at 4-hour intervals for 24 hours after finishing CPB. In addition, laboratory tests including biochemistry, coagulation, and blood cell count were performed when patient was admitted to the ICU and 6 hours later. Chest radiography at the admission in ICU was used to assess the correct position of the central venous line. Extubation occurred in the ICU using the following criteria: Tobin index (respiratory rate [spontaneous]/tidal volume [liters]) less than 105; $\mathrm{PaO}_{2}$ greater than $60 \mathrm{~mm} \mathrm{Hg}$; fraction of inspired oxygen $\left(\mathrm{FlO}_{2}\right)$ less than 0.4 ; continuous positive airway pressure less than 5 mbar, $\mathrm{PaCO}_{2}$ less than $50 \mathrm{~mm} \mathrm{Hg}$, and arterial $\mathrm{pH}$ greater than 7.35 .

\subsection{Study variables}

\subsubsection{Outcome variables}

The primary outcome variable was any degree of AKI, measured by RIFLE criteria (Risk, Injury and Failure).

\subsubsection{Independent variables}

Preoperative, intraoperative, and postoperative potential risk factors for AKI and parameters at admission in the ICU (see below) were defined as independent variables. The preoperative risk for operative mortality was evaluated by additive and logistic EuroSCORE 1.

\subsubsection{Data collection}

The following data were recorded prospectively for all patients:

The following preoperative characteristics were documented: age, sex, weight, height, body surface area, coexisting disease or condition (systemic and pulmonary arterial hypertension, atrial fibrillation, diabetes mellitus, hepatic disease, chronic obstructive pulmonary disease, and immunosuppression), previous cardiac surgery, preoperative creatinine, left ventricular ejection fraction, type of surgery (isolated valve surgery, $\mathrm{CABG}$, combined valve + $\mathrm{CABG}$, and surgery of the ascending aorta, emergent surgery), and EuroSCORE (standard and logistic).

Intraoperative characteristics are as follows: $\mathrm{CPB}$ time and aortic cross-clamp time, lactate, and C-reactive protein.

Postoperative characteristics are as follows: duration of mechanical ventilation, time to extubation, reintubation, tracheotomy, use of vasopressor drugs, red blood cell transfusion, acute renal failure, 
reintervention, intraaortic balloon pump, pneumonia, mean ICU and inhospital stay, and mortality.

\subsubsection{Parameters at admission to the ICU}

The following data were collected immediately at the admission in the ICU: $\mathrm{pH}$, bicarbonate $\left(\mathrm{HCO}_{3}\right)$, partial pressure of carbonic anhydride $\left(\mathrm{PaCO}_{2}\right), \mathrm{PaO}_{2} / \mathrm{FiO}_{2}$ ratio, $\mathrm{CO}_{2}$ arteriovenous difference, central venous oxygen saturation $\left(\mathrm{ScvO}_{2}\right)$, leukocyte, C-reactive protein, procalcitonin, lactate, glucose, creatinine, hematocrit, sodium $(\mathrm{Na})$, potassium $(\mathrm{K})$, troponin $\mathrm{T}$, creatine kinase-MB, international normalized ratio (INR), activated partial thromboplastin time ratio, and platelet count.

Core temperature, heart rate, mean arterial pressure, urinary output, amount of fluids infused in the first 24 hours, and fluid balance were collected. In all cases, hemodynamic parameters (heart rate and mean arterial pressure) were registered 30 minutes after admission to the ICU and once the patient had reached hemodynamic stability.

\subsection{Statistical analysis}

Statistical analyses were performed using the IBM Statistical Package for Social Sciences (IBM SPSS) version 20. Differences between the 2 groups were examined using the Student $t$ test for continuous variables and Pearson $\chi^{2}$ test for categorical data. $P<.05$ was considered significant for all statistical tests.

To assess the associations between the preoperative, intraoperative, and postoperative parameters and AKI after cardiac operations, all variables determined on preoperative and intraoperative period and on admission to the ICU and which were significant in the univariate analysis were subsequently tested for accuracy with a receiver operating characteristic (ROC) curve analysis, with the area under the curve (AUC) as a measurement of accuracy, according to the methodology used and validated in other studies [14]

The variables with areas under the ROC curve less than 0.5 were transformed into their inverses, to observe the same classification criteria with all the variables [15]. The variables with the best AUC values (AUC $>0.7$ ) were used in a subsequent multivariable logistic analysis. To adjust the coefficients with greater precision (to avoid losing cases with the rest of the variables that were not significant), the logistic regression was repeated with the variables that had been significant in the previous analysis. This logistic regression model was tested for calibration with a Hosmer-Lemeshow $\chi^{2}$, and the possible multicollinearity of the model was checked with an analysis of tolerance and inflation statistics.

The above model was subsequently translated into an AKI risk score, developed based on the respective weights of the significant variables (based on their regression coefficients). Using the B coefficients of the logistic regression, the logit function $(\mathrm{Z})$ was constructed, and the $\mathrm{Z}$ values and each one of the products BiXi were saved as new variables in the database $(\mathrm{Z}=\mathrm{B} 0+\mathrm{B} 1 \mathrm{X} 1+\mathrm{B} 2 \mathrm{X} 2+\ldots+\mathrm{BnXn})$.

Receiver operating characteristic curves were generated for each of these new variables and used to identify the cutoff points that defined the ranges of scores for AKI for each predictor. The cutoff points of the ROC curves for scores (BiXi) were correlated with the corresponding values of the original variables. The corresponding BiXi average value was allocated as a point score to each resultant range of values of the original variable Xi (predictor) within that particular grouping. The sum of weighted risk scores was then calculated for each patient. Initial risk categories for AKI were calculated from the cutoff points defined by the ROC curve obtained with the overall point score.

Differences between predicted and observed AKI rates were explored for different risk classes, determined by their cutoff points on the ROC curve, by comparing predicted/observed event rates with $95 \%$ confidence intervals (CIs).

\section{Results}

\subsection{Patient characteristics}

During the duration of the study, a total of 909 adult patients were operated on at the cardiac surgery center concerned. Twelve patients died in the operating theater. Seventeen patients died during the first 24 hours of admission in the ICU. Seventy patients were scheduled for off-pump cardiac surgery. A total of 810 patients were enrolled in the study, developing AKI 137 (16.9\%). In addition, in the validation cohort, 117 patients (15.7\%) developed AKI.

\subsection{Differences between AKI and NON-AKI patients}

Patients who did not have AKI were younger and had a significant higher operative risk as demonstrated by a higher EuroSCORE 1 values (Table 1). The AKI group more frequently showed a medical history of previous atrial fibrillation, diabetes, pulmonary hypertension, poor mobility, recent myocardial infarction, endocarditis, and arteriopathy. Furthermore, they underwent emergency surgery or redo interventions more often. The NON-AKI patients were treated more frequently with statins and $\beta$-blockers. Acute kidney injury patients also showed a lower left ventricular ejection fraction and had longer CPB and aortic

Table 1

Patient population profile (AKI and NON-AKI)

\begin{tabular}{|c|c|c|c|}
\hline Variable & NON-AKI, 673 (83.1\%) & AKI, 137 (16.9\%) & $P$ \\
\hline \multicolumn{4}{|l|}{ Perioperative factors } \\
\hline Age $(y)$ & $66.2 \pm 10.9$ & $70.0 \pm 10.3$ & $<.001$ \\
\hline Weight (kg) & $72.9 \pm 15.7$ & $70.7 \pm 10.3$ & .04 \\
\hline Height (cm) & $163.4 \pm 9.3$ & $161.8 \pm 7.5$ & .03 \\
\hline $\operatorname{LVEF}(\%)$ & $57.3 \pm 10.9$ & $51.1 \pm 13.9$ & $<.001$ \\
\hline Logistic EuroSCORE 1 (\%) & $4.6 \pm 4.9$ & $12.9 \pm 13.2$ & $<.001$ \\
\hline Additive EuroSCORE 1 (points) & $4.21 \pm 2.57$ & $7.61 \pm 3.33$ & $<.001$ \\
\hline Hypertension & $363(53.9)$ & $83(60.6)$ & .15 \\
\hline Atrial fibrillation & $127(18.9)$ & $48(27.4)$ & $<.001$ \\
\hline Previous cardiac surgery & $63(9.4)$ & $13(17.1)$ & .96 \\
\hline Type 2 diabetes mellitus & $97(14.4)$ & $33(24.1)$ & .005 \\
\hline Smoking & $93(13.8)$ & $14(10.2)$ & .26 \\
\hline Alcohol & $43(6.4)$ & $7(5.1)$ & .57 \\
\hline Emergent surgery & $34(5.1)$ & $37(27.0)$ & $<.001$ \\
\hline Statins & $362(53.8)$ & $61(44.5)$ & .048 \\
\hline$\beta$-Blockers & $384(57.1)$ & $43(31.4)$ & $<.001$ \\
\hline Poor mobility & $22(3.3)$ & $13(9.5)$ & .001 \\
\hline Pulmonary hypertension >60 & $37(5.5)$ & 19 (13.9) & $<.001$ \\
\hline Endocarditis & $15(2.2)$ & $7(5.1)$ & .06 \\
\hline Recent myocardial infarction & $66(9.8)$ & $33(24.1)$ & $<.001$ \\
\hline Arteriopathy & $55(8.2)$ & 19 (13.9) & .03 \\
\hline Previous cardiac surgery & $29(4.3)$ & $12(8.8)$ & .03 \\
\hline Critically ill & $10(1.5)$ & $25(18.2)$ & $<.001$ \\
\hline Valve replacement & $413(61.4)$ & $84(61.3)$ & .991 \\
\hline CABG & $334(49.6)$ & $64(46.7)$ & .53 \\
\hline Valve + CABG & $91(13.5)$ & $27(19.7)$ & .06 \\
\hline \multicolumn{4}{|l|}{ Intraoperative factors } \\
\hline Total CPB time (min) & $107.8 \pm 37.2$ & $143.4 \pm 56.5$ & $<.001$ \\
\hline Aortic cross-clamp time (min) & $78.6 \pm 31.4$ & $105.1 \pm 46.5$ & $<.001$ \\
\hline Lactate during surgery $(\mathrm{mg} / \mathrm{dL})$ & $19.4 \pm 8.3$ & $21.3 \pm 8.6$ & .24 \\
\hline CRP during surgery $(\mathrm{mg} / \mathrm{L})$ & $9.3 \pm 27.8$ & $17.5 \pm 40.3$ & .13 \\
\hline \multicolumn{4}{|l|}{ Postoperative factors } \\
\hline Sepsis & $11(1.6)$ & $14(10.2)$ & $<.001$ \\
\hline Septic shock & $8(1.2)$ & $20(14.6)$ & $<.001$ \\
\hline Reintubation & $10(1.5)$ & $23(16.8)$ & $<.001$ \\
\hline Multiple transfusion & $219(32.5)$ & $34(24.8)$ & .075 \\
\hline RRT & $0(0.0)$ & 19 (13.9) & $<.001$ \\
\hline Tracheostomy & $16(2.4)$ & $22(16.9)$ & $<.001$ \\
\hline Reintervention & $15(2.2)$ & $12(8.8)$ & $<.001$ \\
\hline IABP & $15(2.2)$ & $29(21.2)$ & $<.001$ \\
\hline Cardiac arrest & $15(2.2)$ & $41(29.9)$ & $<.001$ \\
\hline ICU readmission & $9(1.3)$ & $15(10.9)$ & $<.001$ \\
\hline Pneumonia & $19(2.8)$ & $22(16.1)$ & $<.001$ \\
\hline
\end{tabular}

Values are expressed as numbers ( $\mathrm{n})$, percentages (\%), and means \pm SD. LVEF indicates left ventricular ejection fraction; CRP, C-reactive protein; IABP, intraaortic balloon pump. 
cross-clamping time. During the postoperative period, complications such as sepsis, reintervention, tracheostomy, or pneumonia were statistically significant higher in the AKI group, with longer inhospital and ICU length of stay. Finally, AKI patients had a significant higher mortality as demonstrated by Kaplan-Meier analysis (Fig. 1).

\subsection{Predictors of CSA-AKI}

Patients with AKI and without AKI differed in all parameters measured on their admission to the ICU except in glucose, sodium, platelet count, and arterial carbon dioxide partial pressure (Table 2). An ROC analysis was performed for each one of these variables identified in the univariate analyses, with estimation of the AUC and 95\% CI. The variables with the best AUC were standard EuroSCORE 1, CPB time, and postoperative lactate (ICU lactate), bicarbonate, procalcitonin, creatinine (ICU creatinine), troponin T, mean arterial pressure, $\mathrm{ScvO}_{2}$, and INR. These 10 variables were subsequently entered into a multivariate logistic model (forward stepwise). In this analysis, 4 variables (ICU creatinine, ICU lactate, standard EuroSCORE, and CPB time) were confirmed to be independent predictors of AKI. We performed the multivariate logistic model again including only these 4 variables.

Acute kidney injury risk (Table 3) was directly correlated with ICU creatinine (odds ratio [OR], 9.66; 95\% CI, 4.77-19.56), EuroSCORE 1 (OR, 1.40; CI, 1.29-1.52), ICU lactate (OR, 1.03; CI, 1.01-1.04), and CPB time (OR, 1.01; CI, 1.01-1.02). This multivariate model was well calibrated (Table 3; see Hosmer-Lemeshow value).

\subsection{Development of an AKI score: The CRATE score}

The above model, with these 4 variables, was subsequently translated into an AKI risk score: the CRATE score (Table 4). The AKI risk score was developed based on the respective weights of the significant variables (based on their regression coefficients; Table 3), and cutoff points were established by analyzing the ROC curve coordinates for mortality risk according to the products BiXi. The cutoff points of the ROC curves define the different points scale for each of the BiXi variables (Fig. 2). We assigned the median value of the corresponding BiXi value in that same range (p.e. "Lactate score" = 13 in the above "Lactate" range for $\mathrm{BiXi}=0.031^{*}$ ["Lactate"]) to the interval defined by the minimum-
Table 2

Parameters at admission to the ICU in AKI and NON-AKI patients

\begin{tabular}{|c|c|c|c|}
\hline $\mathrm{n}=810$ & NON-AKI, 673 (83.1\%) & AKI, 137 (16.9\%) & $P$ \\
\hline $\mathrm{pH}$ & $7.38 \pm 0.06$ & $7.34 \pm 0.08$ & $<.001$ \\
\hline Bicarbonate (mEq/L) & $24.6 \pm 3.2$ & $22.7 \pm 3.4$ & $<.001$ \\
\hline Lactate $(\mathrm{mg} / \mathrm{dL})$ & $28.1 \pm 13.1$ & $48.9 \pm 33.3$ & $<.001$ \\
\hline Procalcitonin (ng/mL) & $0.6 \pm 3.8$ & $2.1 \pm 7.1$ & .01 \\
\hline Core temperature $\left({ }^{\circ} \mathrm{C}\right)$ & $36.6 \pm 0.8$ & $36.9 \pm 1.1$ & $<.001$ \\
\hline Creatinine $(\mathrm{mg} / \mathrm{dL})$ & $0.96 \pm 0.27$ & $1.35 \pm 0.47$ & $<.001$ \\
\hline Heart rate (beats per minute) & $92.2 \pm 17.4$ & $100.8 \pm 19.4$ & $<.001$ \\
\hline Glucose $(\mathrm{mg} / \mathrm{dL})$ & $181.6 \pm 53.9$ & $189.3 \pm 71.9$ & .23 \\
\hline Leukocyte count (cells/mm³) & $10315 \pm 6526$ & $12734 \pm 5889$ & $<.001$ \\
\hline Hematocrit (\%) & $29.1 \pm 4.4$ & $28.2 \pm 4.2$ & .02 \\
\hline $\mathrm{Na}(\mathrm{mmol} / \mathrm{L})$ & $140.2 \pm 8.2$ & $141.03 \pm 4.2$ & .22 \\
\hline $\mathrm{K}(\mathrm{mmol} / \mathrm{L})$ & $3.9 \pm 0.6$ & $4.1 \pm 0.7$ & $<.001$ \\
\hline C-reactive protein (mg/L) & $12.7 \pm 34.02$ & $26.5 \pm 48.9$ & .002 \\
\hline Troponin $\mathrm{T}(\mu \mathrm{g} / \mathrm{L})$ & $0.76 \pm 1.50$ & $1.89 \pm 2.73$ & $<.001$ \\
\hline CK-MB (ng/mL) & $355.5 \pm 285.6$ & $219.9 \pm 274.1$ & $<.001$ \\
\hline $\mathrm{MAP}(\mathrm{mm} \mathrm{Hg})$ & $73.1 \pm 12.9$ & $60.9 \pm 13.8$ & $<.001$ \\
\hline INR (range) & $1.53 \pm 0.24$ & $1.80 \pm 0.50$ & $<.001$ \\
\hline aPTT (range) & $1.21 \pm 0.24$ & $1.46 \pm 0.49$ & $<.001$ \\
\hline Platelet count (cells $/ \mathrm{mm}^{3}$ ) & $118426 \pm 44235$ & $115810 \pm 56590$ & .55 \\
\hline $\mathrm{ScvO} 2(\%)$ & $74.3 \pm 8.10$ & $66.02 \pm 9.78$ & $<.001$ \\
\hline $\mathrm{PaO}_{2} / \mathrm{FlO}_{2}$ (range) & $248 \pm 72.7$ & $231 \pm 64.9$ & .013 \\
\hline $\mathrm{aCO}_{2}(\mathrm{~mm} \mathrm{Hg})$ & $40.9 \pm 6.13$ & $41.3 \pm 6.47$ & .51 \\
\hline $\mathrm{vCO}_{2}(\mathrm{~mm} \mathrm{Hg})$ & $46.9 \pm 6.4$ & $48.9 \pm 6.81$ & .001 \\
\hline Delta $\mathrm{CO}_{2}(\mathrm{~mm} \mathrm{Hg})$ & $5.9 \pm 2.9$ & $7.6 \pm 3.4$ & $<.001$ \\
\hline Inhospital stay (d) & $20.3 \pm 15.4$ & $35.5 \pm 31.1$ & $<.001$ \\
\hline ICU stay $(d)$ & $3.6 \pm 8.6$ & $13.3 \pm 18.0$ & $<.001$ \\
\hline
\end{tabular}

Values are expressed as means \pm SD. CK-MB indicates creatine kinase-MB; MAP, mean arterial pressure; aPTTr, activated partial thromboplastin time ratio; $\mathrm{aCO}_{2}$, arterial carbon dioxide partial pressure; $\mathrm{vCO}_{2}$, venous carbon dioxide partial pressure; Delta $\mathrm{CO}_{2}$, venousarterial carbon dioxide difference; $\mathrm{TnT}$, troponin $\mathrm{T}$.

maximum of each original variable in that group (p.e. "Lactate" 36.9 to $51.0 \mathrm{mg} / \mathrm{dL}$ ).

The new scale was applied to the database, and a total point score was calculated: CRATE (total score) $=$ CReatinine score + lactic Acid score + CPB Time score + EuroSCORE 1 score. The CRATE score created with these 4 variables was tested for significance, accuracy, and calibration using a logistic regression model with Hosmer-Lemeshow $\chi^{2}$ and ROC analysis (Table 5). The CRATE score was significantly $(P<.001)$
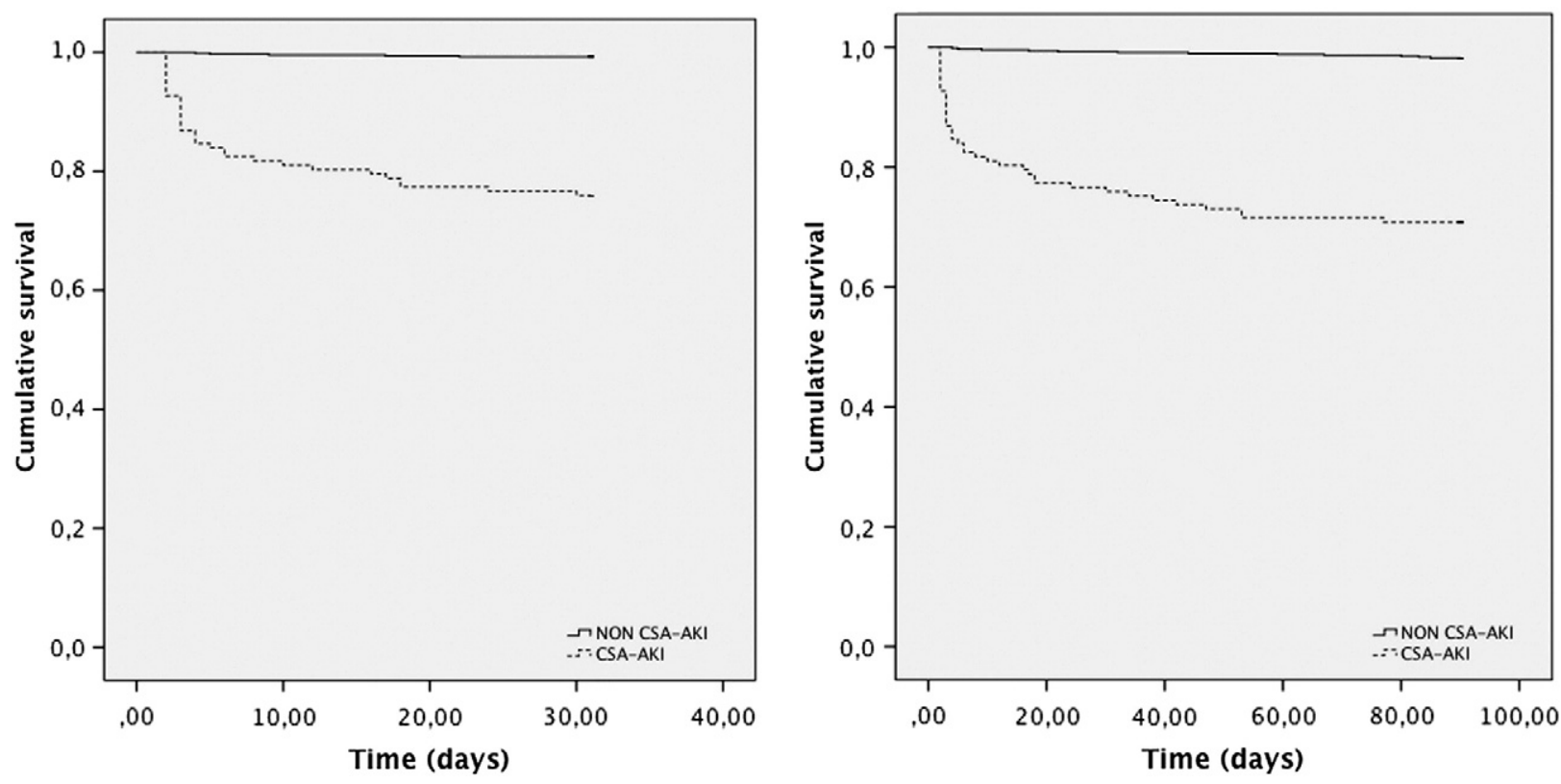

Fig. 1. Kaplan-Meier analysis of 30- and 90-day inhospital survival based on CSA-AKI. 
Table 3

Logistic regression model: risk factors for CSA-AKI

\begin{tabular}{lrlllr}
\hline & \multicolumn{1}{l}{ B } & SE & \multicolumn{1}{l}{$P$} & \multicolumn{1}{l}{ OR } & \multicolumn{1}{l}{$95 \% \mathrm{CI}$} \\
\hline Constant & -8.422 & 0.626 & $<.001$ & & \\
Additive EuroSCORE (points) & 0.336 & 0.042 & $<.001$ & 1.400 & $1.288-1.521$ \\
CPB time (min) & 0.010 & 0.003 & $<.001$ & 1.010 & $1.005-1.016$ \\
Lactate (mmol/L) & 0.031 & 0.007 & $<.001$ & 1.031 & $1.018-1.045$ \\
Creatinine (mg/dL) & 2.268 & 0.360 & $<.001$ & 9.658 & $4.769-19.558$ \\
\hline
\end{tabular}

Hosmer-Lemeshow $\chi^{2}=10.46 ; P=.23$. Multicollinearity diagnostics: EuroSCORE, 0.91 tolerance; CPB time, 0.81 tolerance; lactate, 0.78 tolerance; and creatinine, 0.87 tolerance. $\mathrm{B}$ indicates regression coefficient.

correlated with AKI and demonstrated very good calibration (HosmerLemeshow test $\chi^{2}=11.87 ; P=.16$; Table 5 ). The accuracy was moderately good, with an AUC of 0.89 (95\% CI, 0.85-0.92) (Fig. 3).

\subsubsection{Cardiac surgery-associated acute kidney injury risk categories}

The probability of AKI was calculated for each of the groupings determined by the cutoff points of the ROC curve for the total score (Fig. 2). On this basis, patients were classified into 6 risk categories, which were later regrouped into 5: very low ( $<50$ points), low (50-70 points), moderate (70-80 points), high ( $80-90)$, and very high ( $>90$ points). The observed AKI was $1.69 \%, 8.84 \%, 32.33 \%, 58.66 \%$, and $84.95 \%$, respectively. The percent probability $(95 \% \mathrm{CI})$ for these categories was 1.69 (1.61-1.77), 8.84 (8.3-9.37), 32.33 (30.57-34.09), 58.66 (56.84-60.48), and 84.95 (82.82-87.07), respectively (Table 4).

\subsubsection{Score validation}

The CRATE score was prospectively validated in a new data set of 741 patients recruited by La Princesa University Hospital, Madrid, and the Clinic University Hospital in Valladolid. Incidence of AKI was $15.7 \%$ $(\mathrm{n}=117)$. The CRATE score retained good discrimination in validation cohort. The area under the ROC curve in the validation cohort was 0.81 (95\% CI, 0.78-0.85) (Fig. 4).

\section{Discussion}

The results of this study confirmed the validity of the proposed hypothesis. An AKI risk score evaluating preoperative, intraoperative, and postoperative variables, including organ function parameters assessed upon admission to the ICU, would allow to predict early and in an accurate manner the risk of developing CSA-AKI when CPB is used in patient with previously normal renal function. The CRATE score is easy to perform bedside and in the first hours of admission in the critical care unit. Furthermore, this model was developed using affordable and widely available variables in the routine care of cardiac surgical patients.

The incidence of AKI in the score development population and in validation cohort was $16.9 \%$ and $15.7 \%$, respectively, which is congruent with previous reports where the incidence of AKI ranged from $5 \%$ to $40 \%[4,9,16]$. Three predictive models for CSA-AKI risk not requiring RRT have been developed so far: NNECDSG [8], MCSPI [7], and AKICS [9]. Unlike our study, NNECDSG and MCSPI only enrolled patients who underwent CABG surgery, so their results could not be applied in other types of cardiac surgery (valve or combined procedures). Furthermore, none of these models used postoperative variables. The appearance of renal injury during this period depends on factors such as previous patient characteristics and several events that take place during surgery (inadequate myocardial protection, complications that prolong surgical duration, coagulation abnormalities, hemodynamic instability, anesthetic-related problems, etc). Thus, it is plausible that intraoperative issues can significantly modify the preoperative stratification risk and increase postoperative risk to higher values than those calculated before surgery. As we have demonstrated in this study, organ function assessment variables obtained in the early postoperative period can estimate the physiologic aggression during CPB. Furthermore, we added preoperative and intraoperative variables to characterize and consider the whole process in the stratification risk procedure.

One of the biggest difficulties in comparing different CSA-AKI scores is the diverse definitions used in the categorization of renal injury for each model; in fact, more than 30 different definitions have been used in the previous literature. As such, the 3 aforementioned score systems use 3 different definitions to diagnose AKI, and this is the main reason that could have affected the lack of generalization of this models [17]. RIFLE [3] criteria were proposed as consensus definition of AKI to standardize it and make the comparisons between different studies easy and reliable. Furthermore, RIFLE criteria have been previously validated in diagnosing renal injury after cardiac surgery. Balancing limitations and strengths of both consensus definitions of AKI, the application of the RIFLE criteria in patients undergoing cardiac surgery may be preferable [12]. CRATE is the first postoperative stratification AKI risk model in cardiac surgery that uses RIFLE in its definition, and therefore, it could be easier to compare with future scores. The variables used in CRATE (ICU-Creatinine, ICU-lactic Acid, cardiopulmonary bypass Time, and EuroSCORE 1) have been previously identified in different studies as predictors of AKI in cardiac surgery context.

Table 4

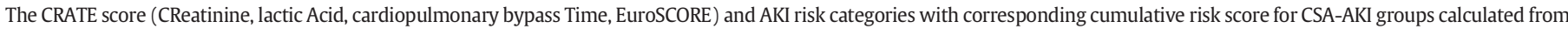
the ROC curve

\begin{tabular}{|c|c|c|c|c|c|}
\hline \multicolumn{2}{|l|}{ Creatinine (mg/dL) } & Creatinine points & \multicolumn{2}{|c|}{ Lactate (mg/dL) } & Lactate points \\
\hline \multicolumn{2}{|l|}{$\leq 0.96$} & 18 & \multicolumn{2}{|l|}{$\leq 17.90$} & 5 \\
\hline $0.97-1.26$ & & 25 & \multicolumn{2}{|c|}{$18.00-23.90$} & 6 \\
\hline $1.27-1.49$ & & 30 & \multicolumn{2}{|c|}{$24.00-36.80$} & 9 \\
\hline \multirow{2}{*}{\multicolumn{2}{|c|}{$\geq 1.50$}} & \multirow[t]{2}{*}{39} & \multicolumn{2}{|c|}{$36.90-51.00$} & 13 \\
\hline & & & \multicolumn{2}{|c|}{$\geq 51.10$} & 21 \\
\hline \multicolumn{2}{|l|}{ CPB time (min) } & $\mathrm{CPB}$ time points & \multicolumn{2}{|c|}{ EuroSCORE (points) } & EuroSCORE points \\
\hline \multicolumn{2}{|l|}{$\leq 81$} & 7 & \multicolumn{2}{|l|}{$\leq 2$} & 7 \\
\hline \multicolumn{2}{|l|}{$82-104$} & 9 & \multicolumn{2}{|l|}{$3.0-4.0$} & 10 \\
\hline \multicolumn{2}{|l|}{$105-138$} & 12 & \multicolumn{2}{|l|}{$5.0-7.0$} & 20 \\
\hline \multicolumn{2}{|l|}{ 139-181 } & 15 & \multicolumn{2}{|l|}{$8.0-10.0$} & 30 \\
\hline \multicolumn{2}{|l|}{$\geq 182$} & 20 & \multicolumn{2}{|l|}{$\geq 11$} & 40 \\
\hline Risk score & Observed n & Predicted n & \% Probability (95\% CI) & AKI (\%) & AKI risk categories \\
\hline$<50$ & 5 & 5 & $1.69(1.61-1.77)$ & 3.6 & Very low \\
\hline $50-70$ & 32 & 32 & $8.84(8.30-9.37)$ & 27 & Low \\
\hline $70-80$ & 27 & 27 & $32.33(30.57-34.09)$ & 46.7 & Moderate \\
\hline $80-90$ & 23 & 23 & $58.66(56.84-60.48)$ & 63.5 & High \\
\hline$>90$ & 50 & 50 & $84.95(82.82-87.07)$ & 100 & Very high \\
\hline
\end{tabular}



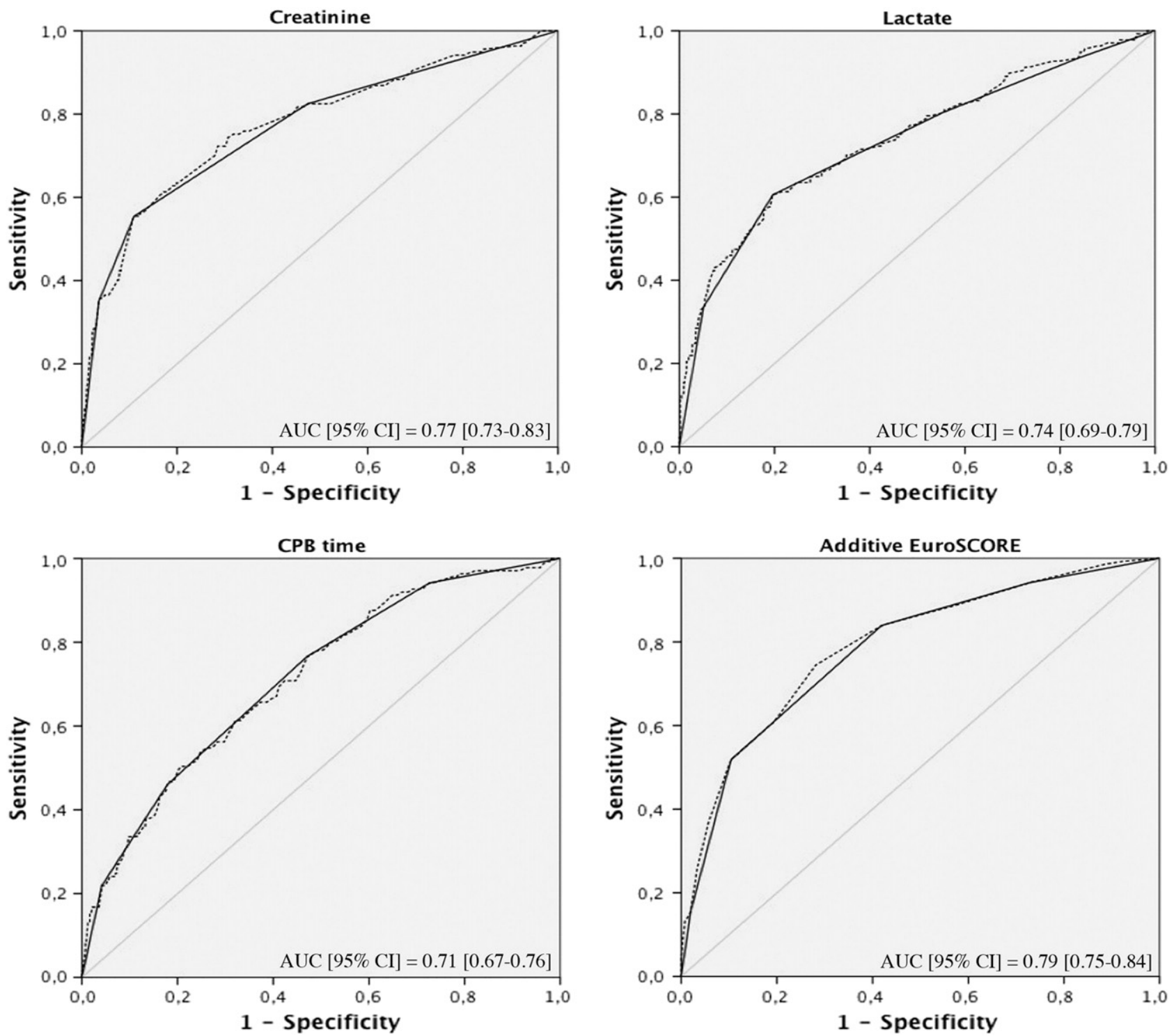

Fig. 2. Receiver operating characteristic curves for individual scores (BiXi) used in CSA-AKI prediction in dotted line (established cutoff points in solid line).

Hyperlactatemia (HL), a well-recognized marker of hypoperfusion, has been associated with organ dysfunction and poor outcome in various clinical settings, including cardiac surgery $[18,19]$. Up to $20 \%$ of patients develop HL during or shortly after CPB, and it frequently persists during the first hours of admission in ICU [18]. The main mechanism of immediate HL after cardiac surgery is an increased rate in lactate production and, to a lesser extent, a diminished clearance ability of the liver. The latter is due to the effect of anesthetics in cellular metabolism and low core temperature during CPB. The use of catecholamines has also been related to HL due to changes induced in oxidative glucose metabolism [19]. Patients with HL during CPB are suffering from a sort of masked circulatory shock, which will exert its deleterious effects on different organs (mainly on renal function) during the early phases of the

Table 5

Development series: predictive function of CRATE score for CSA-AKI risk (logistic regression)

\begin{tabular}{lrllll}
\hline & B & SE & $P$ & OR & $95 \% \mathrm{CI}$ \\
\hline CRATE & 0.114 & 0.009 & $<.0001$ & 1.12 & $1.10-1.14$ \\
Constant & -9.240 & 0.653 & $<.0001$ & 0.00 & \\
\hline
\end{tabular}

Hosmer-Lemeshow test $\chi^{2}=11.87 ; P=.16$. postoperative course [18]. In addition, increased levels of blood lactate have been associated with increased risk of morbidity and mortality and higher incidence of complications including AKI [19].

Creatinine can be measured easily and quickly in blood tests, and it is a specific marker of the kidney function. Furthermore, changes in baseline serum creatinine can reflect changes in glomerular filtration rate [7]. Several studies have shown that increases in serum creatinine after cardiac surgery are related to the development of CSA-AKI in both adult and pediatric population $[2,10]$. In a recent article, Ho et al [11] have demonstrated that small changes in creatinine, measured at ICU admission, can improve prediction of AKI. Accordingly, Shaw et al [20] emphasized that rising serum creatinine levels may be a relevant parameter in the progression to AKI. Thus, even small postoperative increases in serum creatinine may assist in the early prediction of AKI [10].

Previous authors have published that duration of CPB is an independent risk factor for CSA-AKI, establishing a time limit interval between 115 and 180 minutes from which the risk of AKI starts to increase [21,22]. It is plausible that the pathophysiologic changes associated with CPB (systemic inflammatory response, changes in renal vasomotor tone, nonpulsatile blood flow, activation of complement and coagulation pathways, generation of microemboli, and hemolysis) are enhanced as the duration of CPB increases, which subsequently increases 


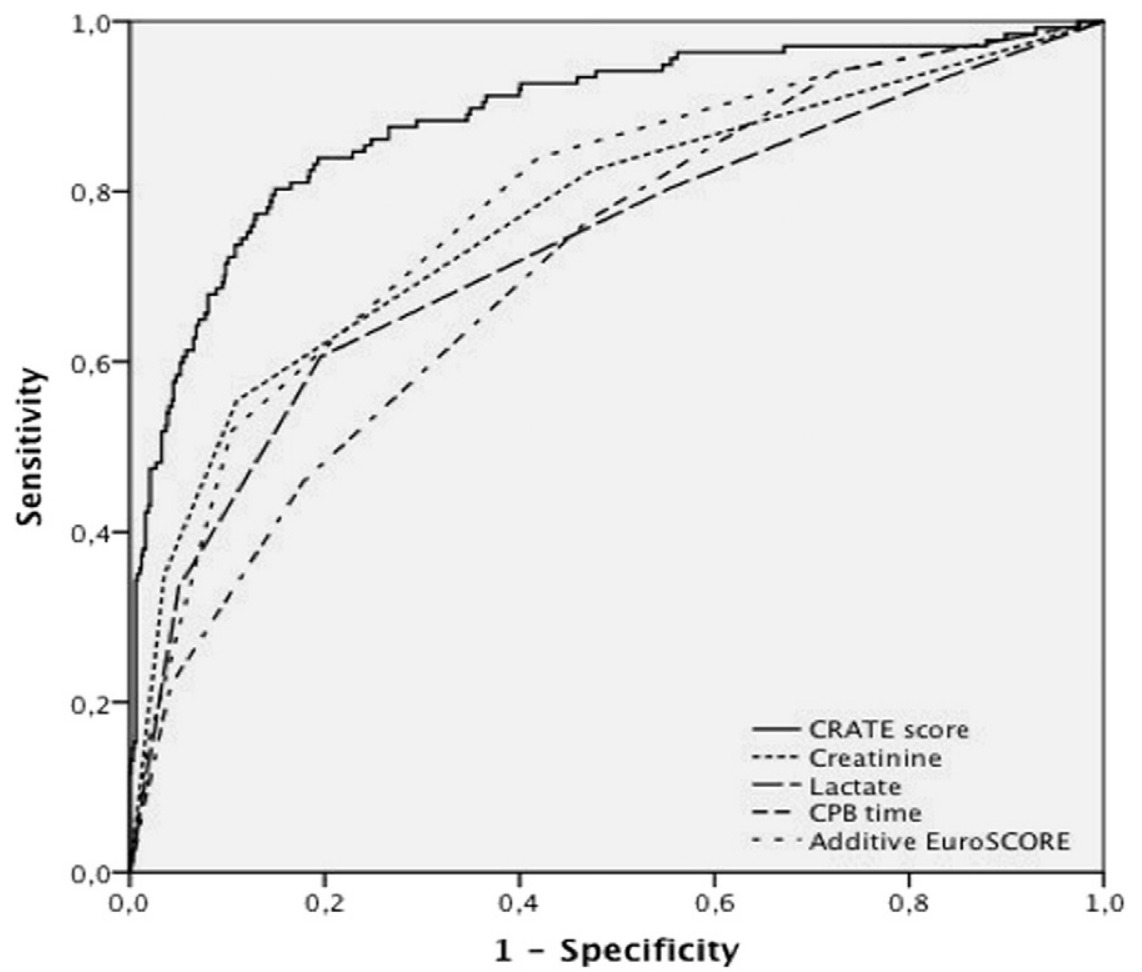

\begin{tabular}{lcccc}
\hline & AUC & SE & Pvalue & $95 \% \mathrm{CI}$ \\
\hline CRATE & 0.887 & 0.017 & $\varangle 0.001$ & $0.852-0.921$ \\
Creatinine & 0.771 & 0.024 & $\varangle 0.001$ & $0.723-0.819$ \\
Lactate & 0.733 & 0.026 & $\varangle 0.001$ & $0.682-0.784$ \\
CPB time & 0.714 & 0.024 & $\varangle 0.001$ & $0.668-0.761$ \\
Ad EuroSCORE & 0.783 & 0.022 & $\varangle 0.001$ & $0.740-0.826$ \\
\hline
\end{tabular}

Fig. 3. CRATE ROC curves, creatinine, lactate, CPB time, and additive EuroSCORE 1 for predicting CSA-AKI.

the risk of developing AKI. In a meta-analysis that included 12466 patients, Kumar et al [22] confirmed that CPB time is an independent risk factor for CSA-AKI, which, in turn, has a significant effect on overall mortality.

In our center, according to the routine practice in Spain and in Europe, we perform EuroSCORE 1 in every patient scheduled for cardiac surgery to assess the preoperative risk. Furthermore, EuroSCORE can be used to predict not only inhospital mortality, for which it was originally designed, but also prolonged length of stay and specific postoperative complications such as renal failure. These outcomes can be predicted accurately using the standard EuroSCORE, which is very simple and easy to calculate [23]. de Moura et al [24] found that there is a statistically significant association between the values of EuroSCORE 1 and the risk of developing AKI in the postoperative period after cardiac surgery. Accordingly, D'Onofrio et al [21] found that patients developing CSA-AKI have more preoperative comorbidities as summarized by the higher EuroSCORE value. Two previous studies have combined EuroSCORE and immunological or biochemical markers with the purpose of implementing the EuroSCORE's predictive ability, resulting in an improvement in the AUC value in both studies [25,26]. Ranucci et al [25] observed 929 patients in a retrospective study and found that, at the admission in ICU, the addition of lactate $(>4 \mathrm{mmol} / \mathrm{L})$ and heart rate $(>120$ beats per minute) to EuroSCORE value, increases the accuracy of this score to stratify mortality risk. Similarly, Kennedy et al [27] have shown that, in conjunction with the logistic EuroSCORE 1, surface expression of $\triangle \mathrm{CD} 99$ and $\triangle \mathrm{CD} 47$ and preoperative migratory response improve the AUC value of EuroSCORE alone in predicting ICU stay. Thus, previous literature justifies that the use of specific parameters along with EuroSCORE can increase the predictive ability oriented to different targets, in our case in AKI scenario. An update of this mortality score has recently been published (EuroSCORE II), so that it would be desirable to carry out a new study that takes into account this modification with the purpose of assessing how this new score fits in CRATE context.

The advent of novel biomarkers of kidney injury has opened a new era of early detection and prognosis prediction for CSA-AKI. Two of the most frequently studied new promising AKI biomarkers to date are neutrophil gelatinase-associated lipocalin (NGAL or lipocalin 2) and interleukin 18 [17]. However, these markers are not widely available in routine practice, and further studies must be developed to extend its use. Neutrophil gelatinase-associated lipocalin is one of the most up-regulated genes and proteins early after AKI and one of the most studied novel biomarkers in the early detection of AKI [16]. Several authors $[27,28]$ have studied the role of NGAL in predicting CSA-AKI and have demonstrated better and earlier predictive power as compared to creatinine. In addition, Haase-Fielitz et al [29] established that the predictive power of NGAL is moderately good, with an AUC of 0.82 to 0.83. In our study, CRATE has a predictive power similar to that of NGAL, with an AUC of 0.89 in the study cohort and 0.81 in the validation cohort. Although we did not perform a cost-benefit study, it is plausible 


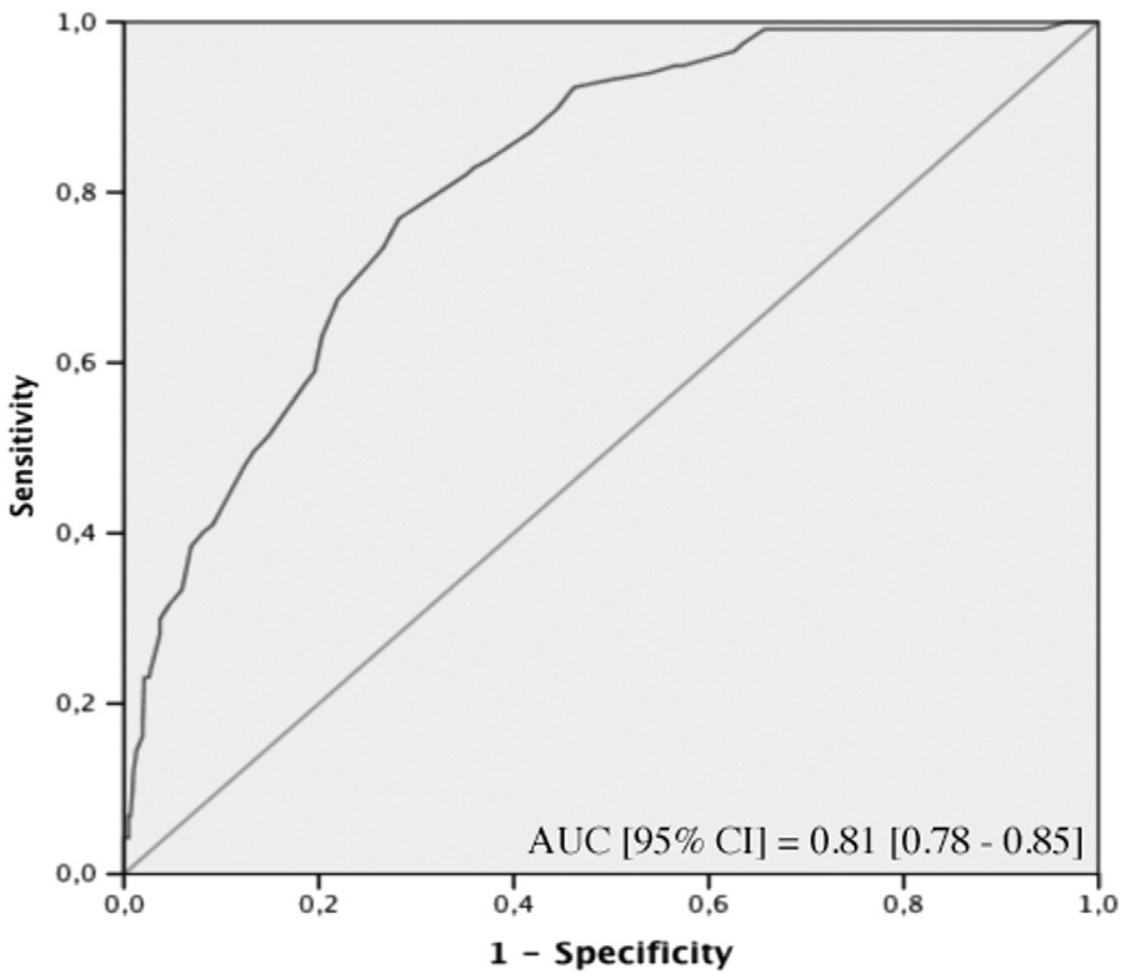

Fig. 4. Receiver operating characteristic curve in the validation cohort.

that applying CRATE in clinical practice will not involve an increase in the cost of the procedure because the variables making up this scale are of routine use in the treatment and follow-up of cardiac surgery patients. Consequently, when NGAL or other biomarkers are unavailable, CRATE could represent a good alternative. Further studies comparing the predictive power of CRATE and NGAL in the same population are necessary. One of the advantages of this study is that an external validation of the scale has been carried out in the same hospital center at a different time, along with a sample of patients from another center, so the results could be generalizable to other populations.

As in previous studies [30], we did not use the RIFLE urine output criteria in patient classification, which can underestimate in a moderate way the incidence of CSA-AKI in our population.

In summary, strategies for the prevention of CSA-AKI must consider identification of high-risk patients, being the early postoperative period the best time to apply a cardiac surgery-specific score because of the influence of intraoperative variables in the clinical course of these patients [9]. CRATE is easy to calculate at the bedside and permits an initial evaluation of AKI risk after a short period (1-2 hours) upon admittance to the ICU, so the clinician could start early interventions to prevent or reduce kidney damage. Furthermore, although we have not carried out a cost analysis, it seems reasonable to think that CRATE is an inexpensive tool because it uses affordable and widely available variables in clinical centers where cardiac surgery is a routine practice.

\section{Funding}

This work was supported in part by a grant from the "Gerencia de Salud, Consejería de Sanidad, Junta de Castilla y Leon" (GRS 463/A/10; code: 18IKMW99) and the Ministry of Health (RD06/0001/0020).

\section{Conflicts of interest}

None declared.

\section{References}

[1] Uchino S, Kellum JA, Bellomo R, Doig GS, Morimatsu H, Morgera S, et al. Beginning and Ending Supportive Therapy for the Kidney (BEST Kidney) Investigators. Acute renal failure in critically ill patients: a multinational, multicenter study. JAMA 2005;294:813-8.

[2] Lassnigg A, Schmidlin D, Mouhieddine M, Bachmann LM, Druml W, Bauer P, et al. Minimal changes of serum creatinine predict prognosis in patients after cardiothoracic surgery: a prospective cohort study. J Am Soc Nephrol 2004;31: 158-65.

[3] Bellomo R, Ronco C, Kellum JA, Mehta RL, Palevsky P, Acute Dialysis Quality Initiative workgroup. Acute renal failure-definition, outcome measures, animal models, fluid therapy and information technology needs: the Second International Consensus Conference of the Acute Dialysis Quality Initiative (ADQI) Group. Crit Care 2004;8: 204-12.

[4] Bellomo R, Auriema S, Fabbri A, D'Onofrio A, Katz N, McCullough PA, et al. The pathophysiology of cardiac surgery-associated acute kidney injury (CSA-AKI). Int J Artif Organs 2008;31:166-78.

[5] Nashef SA, Roques F, Michel P, Gauducheau E, Lemeshow S, Salamon R. European system for cardiac operative risk evaluation (EuroSCORE). Eur J Cardiothorac Surg 1999;16:9-13.

[6] Brown JR, Cochran RP, Dacey LJ, Ross CS, Kunzelman KS, Dunton RF, et al. Perioperative increases in serum creatinine are predictive of increased 90-day mortality after coronary artery bypass graft surgery. Circulation 2006;114:409-13.

[7] Aronson S, Fontes ML, Miao Y, Mangano DT, Investigators of the Multicenter Study of Perioperative Ischemia Research Group, Ischemia Research and Education Foundation. Risk index for perioperative renal dysfunction/failure: critical dependence on pulse pressure hypertension. Circulation 2007;115:733-42.

[8] Brown JR, Cochran RP, Leavitt BJ, Dacey LJ, Ross CS, MacKenzie TA, et al. Multivariable prediction of renal insufficiency developing after cardiac surgery. Circulation 2007; $116: 139-43$

[9] Palomba H, de Castro I, Neto AL, Lage S, Yu L. Acute kidney injury prediction following elective cardiac surgery: AKICS Score. Kidney Int 2007;72:624-31.

[10] Zappitelli M, Bernier PL, Saczkowski RS, Tchervenkov CI, Gottesman R, Dancea A, et al. A small post-operative rise in serum creatinine predicts acute kidney injury in children undergoing cardiac surgery. Kidney Int 2009;76:885-92.

[11] Ho J, Reslerova M, Gali B, Nickerson PW, Rush DN, Sood MM, et al. Serum creatinine measurement immediately after cardiac surgery and prediction of acute kidney injury. Am J Kidney Dis 2012;59:196-201.

[12] Englberger L, Suri RM, Li Z, Casey ET, Daly RC, Dearani JA, et al. Clinical accuracy of RIFLE and acute kidney injury network (AKIN) criteria for acute kidney injury in patients undergoing cardiac surgery. Crit Care 2011;15:R16.

[13] Justice AC, Covinsky KE, Berlin JA. Assessing the generalizability of prognostic information. Ann Intern Med 1999;130:515-24.

[14] Ranucci M, Castelvecchio S, Menicanti L, Frigiola A, Pelissero G. Risk of assessing mortality risk in elective cardiac operations: age, creatinine, ejection fraction, and the law of parsimony. Circulation 2009;119:3053-61. 
[15] Zweig M, Campbell G. Receiver-operating characteristic (ROC) plots: a fundamental evaluation tool in clinical medicine. Clin Chem 1993:39:561-77.

[16] Gaffney AM, Sladen RN. Acute kidney injury in cardiac surgery. Curr Opin Anesthesiol 2015;28:50-9.

[17] Mao H, Katz N, Ariyanon W, Blanca-Martos L, Adýbelli Z, Giuliani A, et al. Cardiac surgery-associated acute kidney injury. Cardiorenal Med 2013;3:178-99.

[18] Ranucci M, De Toffol B, Isgrò G, Romitti F, Conti D, Vicentini M. Hyperlactatemia during cardiopulmonary bypass: determinants and impact on postoperative outcome. Crit Care 2006;10:R167.

[19] Hajjar LA, Almeida JP, Fukushima JT, Rhodes A, Vincent JL, Osawa EA, et al. High lactate levels are predictors of major complications after cardiac surgery. J Thorac Cardiovasc Surg 2013;146:455-60.

[20] Shaw A, Swaminathan M, Stafford-Smith M. Cardiac surgery-associated acute kidney injury: putting together the pieces of the puzzle. Nephron Physiol 2008;109:55-60.

[21] D'Onofrio A, Cruz D, Bolgan I, Auriemma S, Cresce GD, Fabbri A, et al. RIFLE criteria for cardiac surgery-associated acute kidney injury: risk factors and outcomes. Congest Heart Fail 2010;16:32-6.

[22] Kumar AB, Suneja M, Bayman EO, Weide GD, Tarasi M. Association between postoperative acute kidney injury and duration of cardiopulmonary bypass: a metaanalysis. J Cardiothorac Vasc Anesth 2012;25:64-9.

[23] Toumpoulis IK, Anagnostopoulos C, Swistel DG, DeRose Jr JJ. Does EuroSCORE predict length of stay and specific postoperative complications after cardiac surgery? Eur Cardiothorac Surg 2005;27:128-33.
[24] de Moura EB, Bernardes Neto SC, Amorim FF, Viscardi RC. Correlation of the EuroSCORE with the onset of postoperative acute kidney injury in cardiac surgery. Rev Bras Ter Intensiva 2013;25:233-8.

[25] Ranucci M, Ballotta A, Baryshnikova E, Brozzi S, Boncilli A, Surgical and Clinica Outcome Research (SCORE) Group. Intensive care unit admission parameters improve the accuracy of operative mortality predictive models in cardiac surgery. PLoS One 2010;5:e13551.

[26] Kennedy SA, McEllistrem B, Kinsella A, Fan Y, Boyce S, Murphy K, et al. EuroSCORE and neutrophil adhesion molecules predict outcome post-cardiac surgery. Eur J Clin Invest 2012;42:881-90.

[27] Omerika L, Rasic S, Serdarevic N. Importance of determination of urine neutrophil gelatinase associated lipocalin in early detection of acute kidney injury. Coll Antropol 2014;38:161-6.

[28] Kidher E, Harling L, Ashrafian H, Naase H, Chukwuemeka A, Anderson J, et al. Pulse wave velocity and neutrophil gelatinase-associated lipocalin as predictors of acute kidney injury following aortic valve replacement. J Cardiothorac Surg 2014; 9:89.

[29] Haase-Fielitz A, Haase M, Devarajan P. Neutrophil gelatinase-associated lipocalin as a biomarker of acute kidney injury: a critical evaluation of current status. Ann Clin Biochem 2014;51:335-51.

[30] Arnaoutakis GJ, Bihorac A, Martin TD, Hess Jr PJ, Klodell CT, Ejaz AA, et al. RIFLE criteria for acute kidney injury in aortic arch surgery. J Thorac Cardiovasc Surg 2007; 134:1554-60. 